\title{
Effect of Cholesterol on Distribution of Stable, Hydrophobic Perchlorotriphenylmethyl Triethylester Radical Incorporated in Lecithin Liposomal Membranes
}

\author{
Keizo Takeshita, ${ }^{* a}$ Shoko Okazaki, ${ }^{a}$ and Hisao Kansul ${ }^{b}$ \\ ${ }^{a}$ Laboratory of Analytical Chemistry, Faculty of Pharmaceutical Sciences, Sojo University; and ${ }^{b}$ Laboratory of Organic \\ Chemistry, Faculty of Pharmaceutical Sciences, Sojo University; Kumamoto 860-0082, Japan. \\ Received December 22, 2010; accepted February 16, 2011; published online February 21, 2011
}

Perchlorotriphenylmethyl triethylester radical (PTM-TE) is a hydrophobic, stable radical giving a narrow singlet ESR signal with a small satellite signal for ${ }^{13} \mathrm{C}$ in organic solvents. In order to use PTM-TE as a label of liposomal membranes, its manner of incorporation into liposomal membranes was studied. Two components, broad and narrow signals, were observed on the ESR spectrum of PTM-TE incorporated into liposomal membranes composed of egg yolk phosphatidylcholine (egg-PC). The broad signal was increased by the presence of cholesterol in the membranes. The spectral anisotropy of the broad signal was very small as analyzed with oriented planar multilamellar membranes. The narrow signal increased with an increase in temperature in the absence of cholesterol, whereas only a small increase in the signal was observed in the presence of cholesterol. The $g$-value and line width of the narrow signal were very close to those of PTM-TE in mineral oil, whose viscosity is close to the microviscosity in the hydrophobic region of egg-PC membranes. On the other hand, the $g$-value and line width of the broad signal were close to those of solid PTM-TE. These observations indicate that the broad signal observed in liposomes originates from PTM-TE clusters in the membranes. The clusters were dissolved in egg-PC membranes at a PTM-TE/egg-PC molar ratio of less than $\mathbf{0 . 0 1 7}$. However, the clusters were hardly dissolved in the presence of cholesterol.

Key words trityl radical; EPR; liposome; phase separation; drug delivery system

Along with the development and improvement of in vivo magnetic resonance imaging systems such as ESR imaging system and Overhauser enhanced magnetic resonance imaging (OMRI) system, durable organic radicals have been used as imaging agents to probe various physiological status and to trace drugs with these imaging systems. The paramagnetism of durable nitroxyl radicals responds to their redox reactions, and some of them are commercially available. For these reasons, nitroxyl radicals have been conventionally used as labels of drug carriers and probes for in vivo redox status including the generation of reactive oxygen species. ${ }^{1-7)}$ However, the disadvantage of nitroxyl radicals is the loss of their paramagnetism by enzymatic and nonenzymatic reduction in the body. ${ }^{8-11)}$

Triphenylmethyl radicals (trityl radicals) are another family of durable organic radicals. These radicals are resistant to various reducing and oxidizing agents such as ascorbic acid, glutathione, hydrogen peroxide, and iron. ${ }^{12)}$ Some water-soluble trityl radicals such as Oxo63 gave a long ESR relaxation time giving a singlet, a very narrow ESR signal, and the relaxation time relating to the line width was sensitive to the partial pressure of oxygen. Toxicities of the radicals were reportedly low $\left(\mathrm{LD}_{50}, 8 \mathrm{mmol} / \mathrm{kg}\right.$ in mice) ${ }^{13)}$ Therefore, they have been injected intravenously into experimental animals, and in vivo oxygen maps have been imaged with OMRI ${ }^{14}$ and ESR imaging. ${ }^{15)}$ Furthermore, water-soluble trityl radicals were also utilized as probes of superoxide generation ${ }^{12)}$ and $\mathrm{pH}^{16)}$ for ESR spectroscopy and its imaging. In contrast to hydrophilic trityl radicals, only a few reports have described the application of hydrophobic ones. Although perchlorotriphenylmethyl triethylester radical (PTM-TE; Chart 1 ), one of the hydrophobic trityl radicals, also showed a singlet ESR signal, its oxygen response was solvent-dependent. ${ }^{17)}$ The oxygen response of its ESR line width was high in<smiles>CCOC(=O)c1c(Cl)c(Cl)c(C(c2c(Cl)c(Cl)c(C(=O)OCC)c(Cl)c2Cl)c2c(Cl)c(Cl)c(C(=O)OCC)c(Cl)c2Cl)c(Cl)c1Cl</smiles>

Chart 1. Structure of PTM-TE

a highly hydrophobic environment. Therefore, the radical was doped in oxygen-permeable resin chips made of polydimethyl siloxane ${ }^{18}$ or encapsulated into microspheres of hexamethyldisiloxane. ${ }^{19)}$

For the hydrophobic property and stability of PTM-TE, this radical is a potential label to trace drug carriers such as liposomes and lipid microspheres in live animals without using a radioisotope. In order to use PTM-TE as a label of liposomes, knowledge about its manner of incorporation into liposomal membranes is necessary. In this study, multilamellar liposomes composed of either egg yolk phosphatidylcholine (egg-PC) or egg-PC and cholesterol were prepared with PTM-TE. The lateral distribution of PTM-TE in the membranes and the effect of cholesterol on the distribution were analyzed with ESR spectroscopy.

\section{Experimental}

Reagents PTM-TE was synthesized in our laboratory following methods reported in the literature. ${ }^{17)}$ Egg yolk L- $\alpha$-phosphatidylcholine (egg-PC) was purchased from Avanti Polar Lipids, Inc. (Alabaster, AL, U.S.A.), cholesterol and dicetyl phosphate (DCP) from Sigma-Aldrich Chemical Co. (St. Louis, MO, U.S.A.), and mineral oil (aliphatic hydrocarbon; average molecular weight, 385; Ultragrade 15) from BOC Edwards Vacuum Technology 
(Wilmington, MA, U.S.A.).

Preparations of Liposomes and Planar Membranes Multilamellar liposomes were prepared as described by Bangham et al. ${ }^{20)}$ Chloroform solutions of egg-PC $(1.5 \mu \mathrm{mol})$, DCP $(0.1 \mu \mathrm{mol})$, and various amounts of PTMTE or the solutions of egg-PC $(1 \mu \mathrm{mol})$, cholesterol $(0.5 \mu \mathrm{mol})$, DCP $(0.1 \mu \mathrm{mol})$, and various amounts of PTM-TE were mixed separately, and chloroform was evaporated. The lipid films were evacuated and then hydrated with $100 \mu$ l of Dulbecco's phosphate buffered saline (PBS) without $\mathrm{Mg}^{2+}$ and $\mathrm{Ca}^{2+}$ (Life Technologies, Invitrogen; PBS).

Planar multilamellar membranes were prepared as described previously. ${ }^{21)}$ Chloroform solutions of lipids containing PTM-TE were mixed, and the solution was transferred onto a glass plate $(18 \mathrm{~mm} \times 3 \mathrm{~mm}, 0.15 \mathrm{~mm}$ thick). After evaporation of chloroform, lipid films were hydrated with a drop of PBS and covered with another glass plate.

ESR Measurement ESR spectra for liposomes and oriented planar multilamellar membranes were recorded as described previously. ${ }^{21)}$ Liposomal suspension $(14 \mu \mathrm{l})$ was taken into a $100 \mu \mathrm{l}$ disposable micropipette (Drummond Scientific Co., Broomall, PA, U.S.A.), placed in an ESR sample tube, and set in an ESR cavity. Planar multilamellar membranes were placed in an ESR sample tube and set in an ESR cavity so that the orientation of the membrane plane was parallel or perpendicular to the magnetic field. X-Band ESR spectra were recorded at $100 \mathrm{kHz}$ field modulation with a JEOL FX100 spectrometer equipped with a temperature controller. $\mathrm{Mn}^{2+}$ was used as an internal standard of the ESR cavity. Spectral simulation was performed with an isotropic simulation system IsoSim/FA ver. 2.10 (JEOL).

\section{Results}

ESR Spectra of PTM-TE Incorporated in Liposomal Membranes Multilamellar liposomes composed of eggPC/DCP/PTM-TE (1.5: 0.1 : 0.05 molar ratio; egg-PC liposomes) and egg-PC/cholesterol/DCP/PTM-TE (1: $0.5: 0.1$ : 0.05 molar ratio; egg-PC/cholesterol liposomes) were prepared, and ESR spectra for the liposomes were recorded at room temperature (about $28^{\circ} \mathrm{C}$ ). As shown in Fig. 1a, ESR spectrum for egg-PC liposomes showed a narrow singlet signal with shoulders as indicated with arrowheads. The peakto-peak line width $\left(\Delta H_{\mathrm{msl}}\right)$ for the narrow signal was $0.151 \mathrm{mT}$. The shoulder was large in the presence of cholesterol in liposomal membranes (Fig. 1b), and the spectrum showed an apparently broad singlet line $\left(\Delta H_{\mathrm{msl}}=0.444 \mathrm{mT}\right)$. This suggests that the shoulder is not mainly originated from ${ }^{13} \mathrm{C}$ nuclei, but rather from ${ }^{12} \mathrm{C}$-PTM-TE in the state other than that of the radical giving a narrow signal.

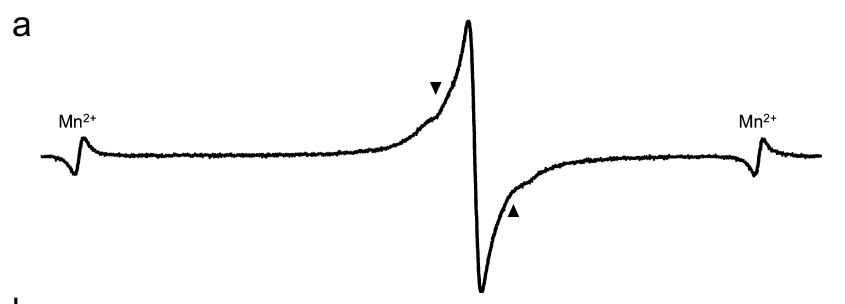

b

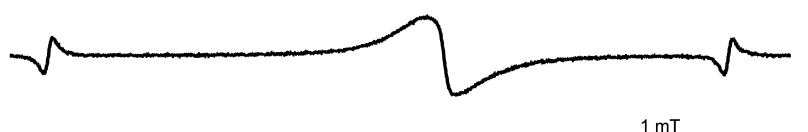

Fig. 1. ESR Spectra of PTM-TE in Egg-PC (a) and Egg-PC/Cholesterol Liposomes (b) at Room Temperature

The egg-PC liposomes composed of $0.05 \mu \mathrm{mol}$ PTM-TE, $1.5 \mu \mathrm{mol}$ egg-PC, and $0.1 \mu \mathrm{mol} \mathrm{DCP}$ and the egg-PC/cholesterol liposomes composed of $0.05 \mu \mathrm{mol} \mathrm{PTM-TE}$, $1.0 \mu \mathrm{mol}$ egg-PC, $0.5 \mu \mathrm{mol}$ cholesterol, and $0.1 \mu \mathrm{mol}$ DCP were prepared in $100 \mu \mathrm{l}$ of PBS. The ESR spectra were recorded at room temperature (about $28^{\circ} \mathrm{C}$ ). Amplitude of the field modulation was $0.08 \mathrm{mT}$. Arrowheads indicate the broad component of the PTM-TE signal.
To examine whether or not orientation of PTM-TE in membranes gives rise to the broad ESR signal, planar multilamellar membranes composed of egg-PC/cholesterol/DCP ( $1: 0.5: 0.1$ molar ratio) were prepared and the spectra were recorded under a magnetic field perpendicular and parallel to the plane of the membrane. As shown in Fig. 2, only a small difference was observed in the shape of these spectra, indicating that the orientation of PTM-TE does not affect the line width of ESR spectrum.

ESR Spectra of PTM-TE in Solutions and Solid PTMTE To characterize ESR spectra of PTM-TE in the liposomal membranes, the ESR spectra of the radical in various solvents were examined. PTM-TE was soluble in organic solvents such as chloroform, $n$-hexane, $n$-octanol, methanol, and mineral oil. The ESR spectra of PTM-TE dissolved in these solvents were composed of narrow singlet line with a small satellite doublet signal that originated from ${ }^{13} \mathrm{C}$ nuclei (Figs. 3a, b), in accordance with the ESR spectrum in the literature in which PTM-TE had been dissolved in dimethylsulfoxide. ${ }^{17)}$ The line widths were $0.149 \mathrm{mT}$ for the chloroform solution and $0.144 \mathrm{mT}$ for the mineral oil solution. These line widths were very close to the value of narrow component of ESR signal for the egg-PC liposomes $(0.151 \mathrm{mT})$. PTM-TE was nearly insoluble in water as demonstrated in Fig. 3c, where no signal was observed in its PBS solution even under saturation.

In contrast to ESR signal for PTM-TE solutions, the signal of solid PTM-TE was composed of a single broad line in accordance with the literature (Fig. $3 \mathrm{~d}$, thick line). ${ }^{17}$ ) The broad signal for egg-PC/cholesterol liposomes was similar to this signal, although the line width of signal for egg-PC/cholesterol liposomes $(0.444 \mathrm{mT})$ was slightly smaller than that of the signal for solid PTM-TE $(0.503 \mathrm{mT})$. This suggests the possibility that the broad signal for the liposomes results from cluster formation of PTM-TE in the membranes. It is noteworthy that the $g$-value of the broad signal for the solid (2.00234) was slightly larger than that of the narrow signal for the mineral oil solution (2.00218), as observed in the superposition of both spectra (Fig. 3d).

Temperature Dependence of ESR Spectra of PTM-TE in Liposomal Membranes It has been reported that increased temperature increased membrane fluidity, and that the increased fluidity, in turn, dissolved phase separation within the membranes. ${ }^{22,23}$ To examine whether or not formation of PTM-TE clusters are involved in the line-broaden-

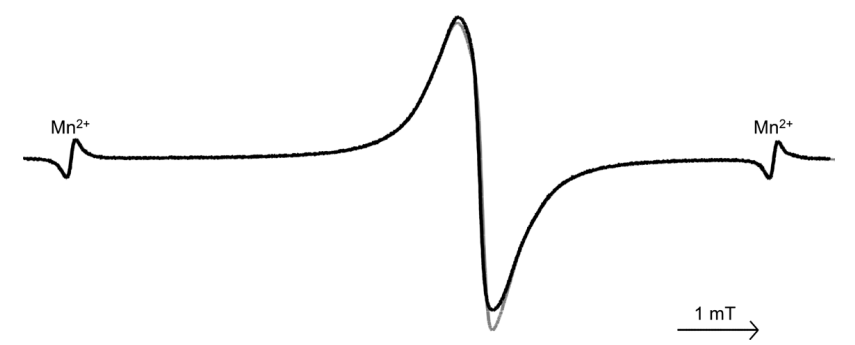

Fig. 2. ESR Spectra of PTM-TE Incorporated into Planar Multilamellar Membranes

Planar multilamellar membranes composed of egg-PC/cholesterol/DCP/PTM-TE $(1: 0.5: 0.1: 0.05$ molar ratio) were prepared and ESR spectra were recorded at room temperature (about $28^{\circ} \mathrm{C}$ ) with the external magnetic field parallel (black line) and perpendicular (gray line) to the membranes. Amplitude of the field modulation was $0.08 \mathrm{mT}$. 


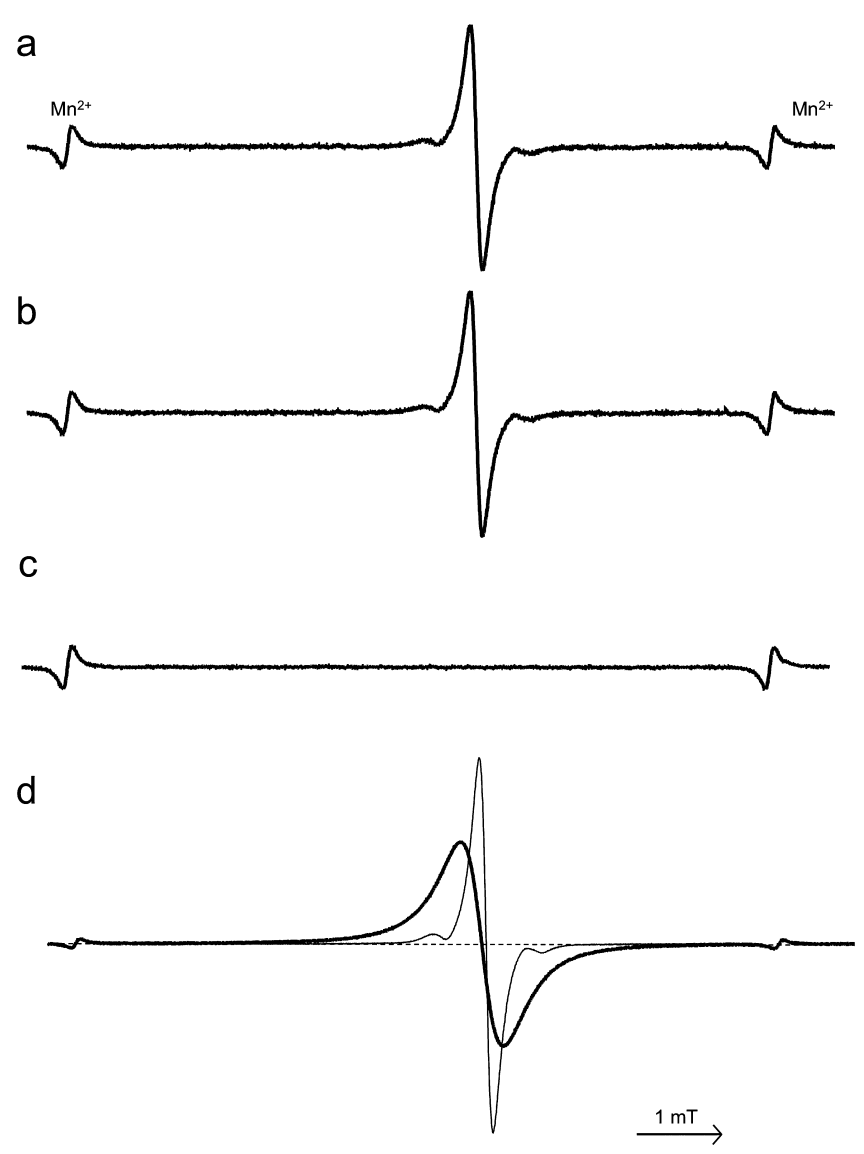

Fig. 3. ESR Spectra of PTM-TE Solutions and Solid PTM-TE

PTM-TE was dissolved in chloroform (a) or mineral oil (b) at a concentration of $0.1 \mathrm{mmol} / 1$, and ESR spectra were recorded. PTM-TE was vigorously mixed with PBS and the aqueous phase was removed. ESR spectrum for the aqueous phase was recorded (b). ESR spectrum for solid PTM-TE (thick line) was superposed on the spectrum for PTM-TE dissolved in mineral oil (thin line) (d). These spectra were recorded at room temperature (about $28^{\circ} \mathrm{C}$ ). Amplitude of the field modulation was $0.016 \mathrm{mT}$ for $\mathrm{a}-\mathrm{c}$ and $0.08 \mathrm{mT}$ for $\mathrm{d}$.

ing, the temperature for ESR measurement was increased. As shown in Fig. 4a and b, the narrow signal was separated and increased in both egg-PC and egg-PC/cholesterol liposomes with an increase in temperature. The increase of the narrow signal was more remarkable in egg-PC liposomes. Those temperature-dependent alterations of the spectra were reversible. The spectrum for egg-PC/cholesterol liposomes was composed of at least two signals at $55^{\circ} \mathrm{C}$ as distinguished by the difference in the line width. As shown in Fig. 5a, the separation of the narrow signal from the broad one was clearer on the shoulder of half-peak at lower field (open arrowhead) than on that of half-peak at higher field (filled arrowhead), indicating that the $g$-value of the broad signal is slightly larger than that of the narrow one. The difference in the $g$ value was confirmed with spectral simulation with a singlet narrow signal $(g=2.0022)$ accompanied by doublet narrow signal and two singlet broad signals $(g=2.0023)$ with difference in their line width (Fig. 5b). These observations support the formation of PTM-TE clusters in the liposomal membranes.

Effects of Molar Ratio of PTM-TE to Lipids on ESR Spectra of PTM-TE When molar ratio of PTM-TE to egg$\mathrm{PC}$ was reduced to one-tenth of the liposomes indicated in Fig. 1a, no broad component was observed in egg-PC liposomes (Fig. 6a). Therefore, effect of molar ratio of PTM- a

b
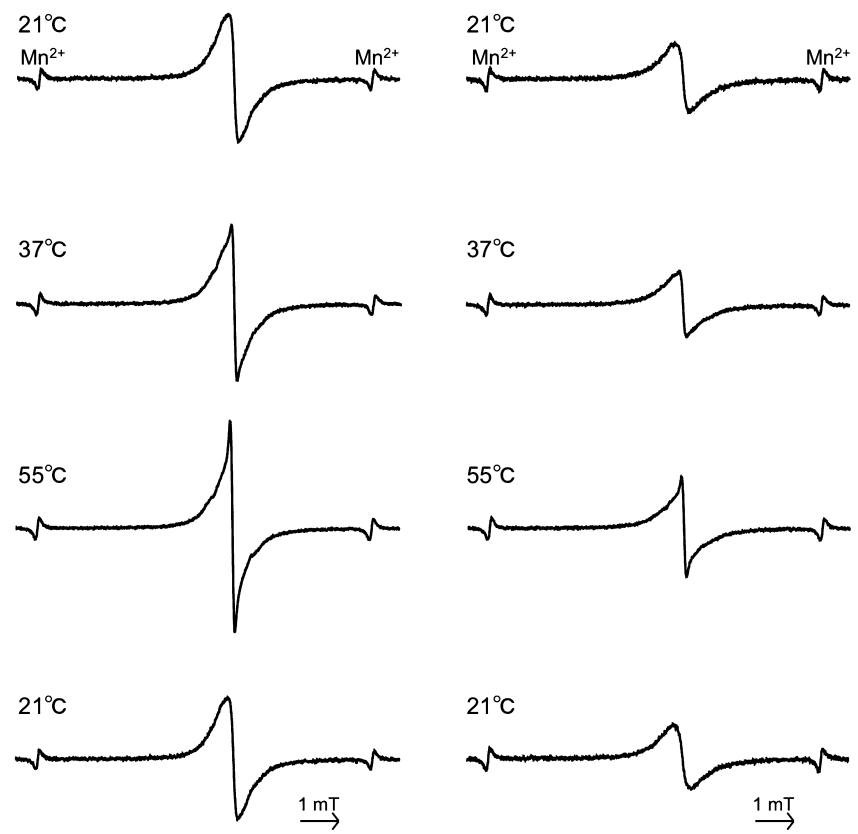

Fig. 4. ESR Spectra of PTM-TE in Egg-PC (a) and Egg-PC/Cholesterol Liposomes (b) at Various Temperatures

The egg-PC and egg-PC/cholesterol liposomes were prepared as mentioned in the legend to Fig. 1. ESR spectra for the liposomes were recorded at various temperatures as indicated at the left hand side of each spectrum. Amplitude of the field modulation was $0.08 \mathrm{mT}$. Temperature for ESR measurement was increased from 21 to $55^{\circ} \mathrm{C}$ and then returned to $21^{\circ} \mathrm{C}$.
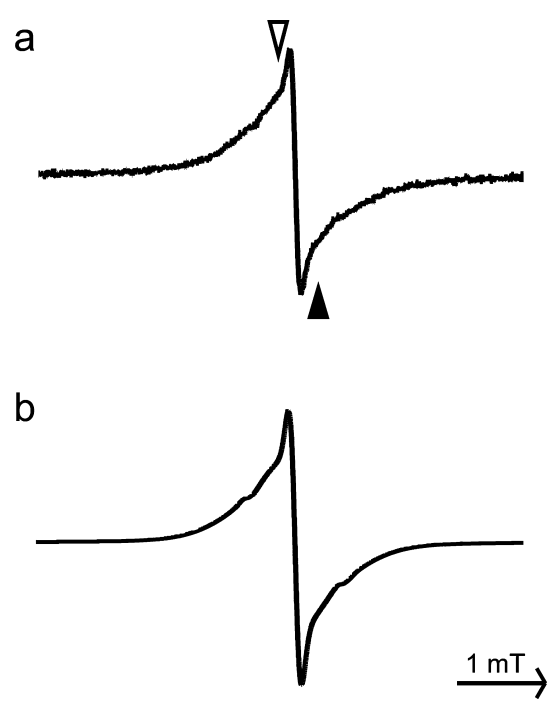

Fig. 5. Experimental and Simulated ESR Spectra for Egg-PC/Cholesterol Liposomes Recorded at $55^{\circ} \mathrm{C}$

Experimental spectrum (a) is the same as that for egg-PC/cholesterol liposomes recorded at $55^{\circ} \mathrm{C}$ in Fig. 4. The broad component on half-peak at lower field (open arrowhead) was clearer than the component on half-peak at higher field (filled arrowhead). The simulated spectrum (b) was composed of four components; a narrow singlet signal ( $g=2.0022, \Delta H_{\mathrm{msl}}=0.129 \mathrm{mT}, 70 \%$ Lorentzian $/ 30 \%$ Gaussian, intensity 20.0 ), a doublet signal $\left(g=2.0022, a^{\mathrm{C} 13}=1.081 \mathrm{mT}, \Delta H_{\mathrm{msl}}=0.129 \mathrm{mT}, 70 \%\right.$ Lorentzian $/ 30 \%$ Gaussian, intensity 1.0), a broad singlet signal $\left(g=2.0023, \Delta H_{\mathrm{msl}}=0.403 \mathrm{mT}, 100 \%\right.$ Lorentzian, intensity 180.0), and a broad singlet signal $\left(g=2.0023, \Delta H_{\mathrm{msl}}=1.000 \mathrm{mT}\right.$, $30 \%$ Lorentzian $/ 70 \%$ Gaussian, intensity 150.0 ).

TE/lipids on the appearance of broad component was examined. Intensity of ESR signal was plotted as a function of concentration of PTM-TE under constant concentration of lipids in the liposomal suspensions (Fig. 6b). The height of 
a

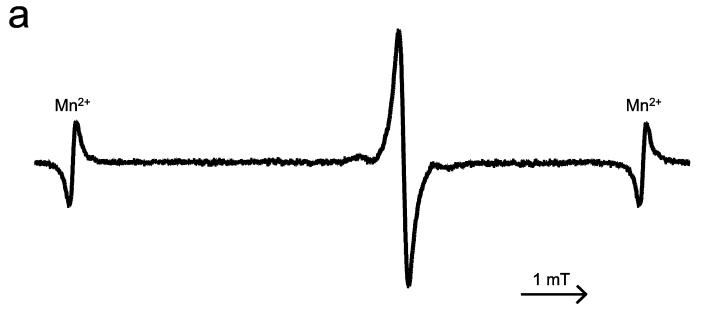

b

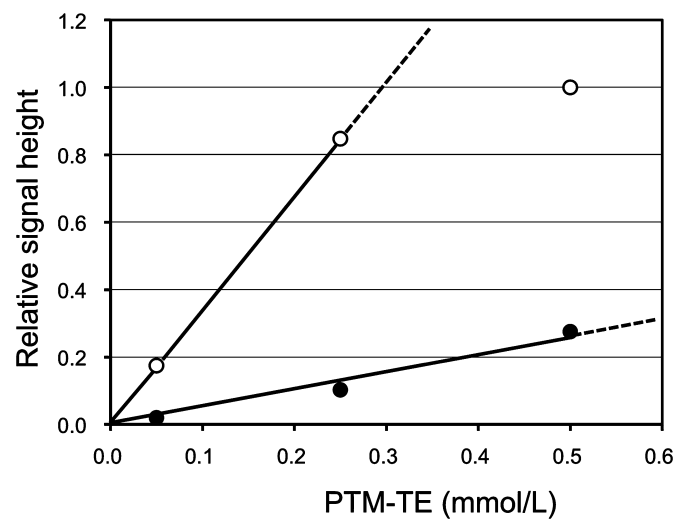

Fig. 6. Effects of PTM-TE/Lipid Ratio on the ESR Spectrum for the Liposomes

(a) Liposomes composed of $0.005 \mu \mathrm{mol}$ PTM-TE, $1.5 \mu \mathrm{mol}$ egg-PC, and $0.1 \mu \mathrm{mol}$ DCP were prepared in $100 \mu \mathrm{l}$ of PBS. ESR spectrum for the liposomes was recorded at room temperature (about $21^{\circ} \mathrm{C}$ ). Amplitude of the field modulation was $0.08 \mathrm{mT}$. (b) The egg-PC liposomes (open circle) and egg-PC/cholesterol liposomes (filled circle) were prepared at various molar ratios of PTM-TE/lipids. The amounts of lipids in the liposomal suspensions were kept constant: $1.5 \mu \mathrm{mol}$ egg-PC and $0.1 \mu \mathrm{mol}$ DCP in $100 \mu \mathrm{l}$ of PBS for egg-PC liposomes and $1.0 \mu \mathrm{mol}$ egg-PC, $0.5 \mu \mathrm{mol}$ cholesterol, an $0.1 \mu \mathrm{mol}$ DCP in $100 \mu \mathrm{l}$ of PBS for egg-PC/cholesterol liposomes. The height of the overall spectrum was plotted against concentration of PTM-TE in the liposomal suspension.

the ESR signal for egg-PC liposomes was on the straight line passing through original point up to $0.25 \mathrm{mmol} / 1$ of PTM-TE. However, the height was lower than this line at $0.5 \mathrm{mmol} / \mathrm{l}$ of PTM-TE because of appearance of broad signal component as observed in Fig. 1a. The observation suggests that clusters of PTM-TE were formed at higher molar ratio of PTM-TE to lipids. The critical concentration of PTM-TE was approximately $0.25 \mathrm{mmol} / \mathrm{ml}$ in the liposomal suspensions, which corresponds to PTM-TE/egg-PC molar ratio $=0.017$. In contrast to egg-PC liposomes, nearly no narrow component was appeared in egg-PC/cholesterol liposomes at any PTM-TE concentrations. The signal height for egg-PC/cholesterol liposomes was lower than that for egg-PC liposomes while the signal increased in proportion to the concentration of PTMTE (Fig. 6b). This may indicate that clusters of PTM-TE are hardly dissolved in the presence of cholesterol in the membranes.

\section{Discussion}

The ESR signal of PTM-TE incorporated in egg-PC liposomal membranes were composed of the narrow and broad components. The presence of cholesterol in the membranes increased the broad component. Both signal components should originate from PTM-TE within the membranes because PTM-TE was nearly insoluble in water. The question was why the broad component appeared in the ESR spectra of PTM-TE in the liposomes. In general, line-broadening of an ESR signal of paramagnetic molecules results from in- complete averaging of $g$-tensor and hyperfine-tensor anisotropies by restricted tumbling motion of the molecules in a viscous environment and from spin-spin interaction with another paramagnetic molecule as usually observed in concentrated solutions of paramagnetic molecules. The line width of the broad component $(0.444 \mathrm{mT})$ was close to that for solid PTM-TE $(0.503 \mathrm{mT})$. An increase in temperature reduced the broad component of the ESR signal for the liposomes. The spectral simulation clarified that the $g$-value for the broad component was slightly larger than that for the narrow component as observed in the ESR spectra of the PTMTE solid and the PTM-TE solution. The broad component was disappeared in egg-PC liposomes, when PTM-TE was diluted in the membranes. These observations strongly suggest that the formation of PTM-TE clusters (PTM-TE-rich domain) in membranes is the origin of the broad signal.

The viscosities of chloroform and the mineral oil are $0.57 \mathrm{mPa} \cdot \mathrm{s}$ and $89 \mathrm{mPa} \cdot \mathrm{s}$, respectively, at $20^{\circ} \mathrm{C}$ (product information of BOC Edwards, Israel). On the other hand, the microviscosity of the hydrophobic region of egg-PC liposomes has been reported to be approximately $80 \mathrm{mPa} \cdot \mathrm{s}$ at $25{ }^{\circ} \mathrm{C},{ }^{24,25)}$ implying that the molecular motion of PTM-TE in mineral oil should be restricted as well as in the hydrophobic region of egg-PC liposomes. The similar spectral line widths for the chloroform solution $(0.149 \mathrm{mT})$ to that for the mineral oil solution $(0.144 \mathrm{mT})$ indicate that the broad component of the ESR signal of PTM-TE in the liposomes $\left(\Delta H_{\mathrm{msl}}>0.4 \mathrm{mT}\right)$ does not result from restricted molecular motion of the radical in the membranes. This conclusion was supported by the observation that the narrow and broad components coexisted even at higher temperature (Fig. 4), because increased temperature should increase the molecular motion of PTM-TE uniformly unless phase separation exists.

The egg-PC membranes are known to be in a liquid crystalline phase at room temperature. It is also known that the hydrophobic molecules are usually distributed homogeneously in phospholipid membranes in liquid crystalline phase. For example, it was reported that spin-labeled androstanol mixed homogeneously with phosphatidylcholine above the gel-liquid crystalline phase transition temperature of the membrane lipid (in liquid crystalline phase) while the spin-labeled sterol formed clusters below this temperature (in gel phase). ${ }^{26)}$ The unusual behavior of PTM-TE is probably attributable to $\mathrm{Cl} \cdots \mathrm{Cl}$ and $\pi \cdots \pi$ interactions between aromatic rings of neighboring radicals. ${ }^{15)}$ Aggregate formations of the derivatives of perchlorotriphenylmethyl radical have been observed in dichloromethane/toluene $(1: 1 \mathrm{v} / \mathrm{v})$ and explained by the above interactions, which had always been observed in crystal structures of the radical derivatives. ${ }^{27)}$ The molecular shape of PTM-TE may also contribute to the phase separation. The derivatives of triarylmethyl radicals including perchlorotriphenylmethyl radicals were reported to be bulky and propeller-shaped ${ }^{28-30)}$ PTM-TE with such a bulky structure may be excluded from membranes to form PTMTE clusters.

In contrast to egg-PC liposomes, PTM-TE easily formed clusters in egg-PC/cholesterol liposomes. PTM-TE clusters were hardly dissolved in egg-PC/cholesterol liposomes, even if the concentration of PTM-TE was decreased in the membranes. Cholesterol is known to harden egg-PC membranes. ${ }^{31)}$ It was reported that cholesterol increased molecu- 


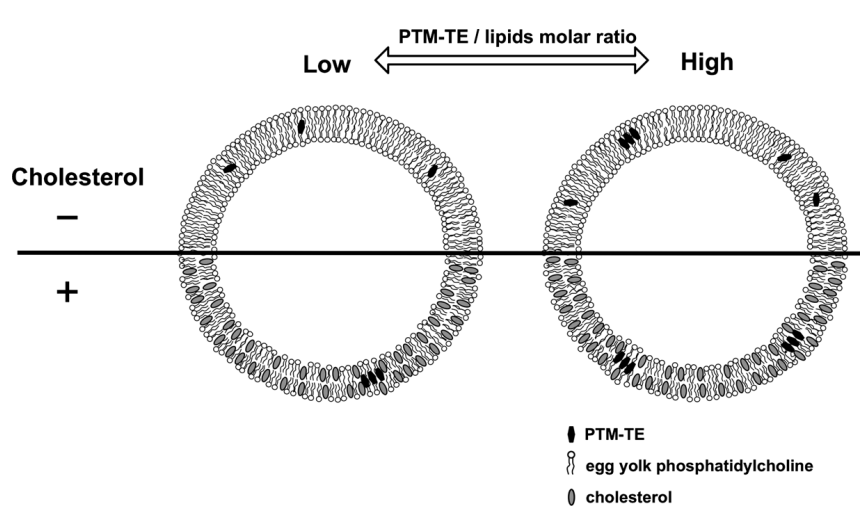

Chart 2. Schematic Illustration of the Effects of Cholesterol and PTM-TE Concentration in Egg-PC Liposomal Membranes on the Distribution of PTM-TE in the Membranes

PTM-TE formed clusters in egg-PC liposomes at high PTM-TE/egg-PC molar ratio, whereas the radical formed clusters in egg-PC/cholesterol liposomes regardless of the molar ratio. The critical PTM-TE/egg-PC molar ratio for the formation of PTM-TE clusters was $c a$. 0.017 in egg-PC liposomes.

lar packing in egg-PC/cholesterol monolayer at the air-water interface. ${ }^{32)}$ The exclusion of bulky PTM-TE molecules from ordered fatty acyl chains of egg-PC is probably liable to occur in lipid membranes. The $\pi \cdots \pi$ interaction between aromatic rings of PTM-TE and cholesterol might also contribute to the formation of a PTM-TE-rich domain.

Because the height of the ESR signal is inversely proportional to the square of the peak-to-peak line width of the signal, the line-broadening caused by clustering of PTM-TE interferes with the sensitive detection of the radical by ESR spectroscopy/imaging and OMRI. Furthermore, phase separation sometimes causes leakage of drugs entrapped in liposomes. ${ }^{33)}$ Therefore, formation of PTM-TE clusters should be avoided when PTM-TE is used as a label to trace drugcarrier liposomes in the body. The present study demonstrated that PTM-TE clusters decreased with a decrease in the molar ratio of PTM-TE to lipids and apparently disappeared at a PTM-TE/egg-PC ratio of $c a$. 0.017, indicating that this ratio should be optimum for labeling. In the present study, DCP was incorporated in the liposomal membranes to stabilize the membranes. It was also confirmed that DCP did not affect distribution of PTM-TE in the membranes (data not shown). Toxicity of PTM-TE is, at present, unclear although toxicity of water-soluble trityl radicals has been reported to be low. ${ }^{13)}$ Transfer of hydrophobic molecules to bio-membranes may induce some effects on the cell functions. Therefore, toxicity also should be examined before application of the liposomes labeled with PTM-TE to pharmacokinetic study of the liposomes.

The conclusion was summarized in Chart 2. PTM-TE was easily incorporated into egg-PC liposomal membranes regardless of the inclusion of cholesterol in the membranes. PTM-TE distributed homogeneously in egg-PC liposomes up to PTM-TE/egg-PC molar ratio of at least 0.017 , while the radical formed clusters at higher PTM-TE/egg-PC molar ratio. On the other hand, PTM-TE apparently existed as only its clusters in the presence of cholesterol in the membranes at room temperature.

Acknowledgment This study was supported by a Grant-in-Aids for Scientific Research (No. 22590052) from the Japan Society for the Promotion of Science.

\section{References}

1) Sano H., Naruse M., Matsumoto K., Oi T., Utsumi H., Free Radic. Biol. Med., 28, 959-969 (2000).

2) Yokoyama H., Lin Y., Itoh O., Ueda Y., Nakajima A., Ogata T., Sato T., Ohya-Nishiguchi H., Kamada H., Free Radic. Biol. Med., 27, 442448 (1999).

3) Kuppusamy P., Afeworki M., Shankar R. A., Coffin D., Krishna M. C., Hahn S. M., Mitchell J. B., Zweier J. L., Cancer Res., 58, 1562-1568 (1998).

4) Takeshita K., Ozawa T., J. Radiat. Res., 45, 373-384 (2004).

5) Takeshita K., Kawaguchi K., Fujii-Aikawa K., Ueno M., Okazaki S., Ono M., Krishna M. C., Kuppusamy P., Ozawa T., Ikota N., Cancer Res., 70, 4133-4140 (2010).

6) Utsumi H., Yamada K., Arch. Biochem. Biophys., 416, 1-8 (2003).

7) Yamato M., Egashira T., Utsumi H., Free Radic. Biol. Med., 35, 1619-1631 (2003).

8) Eriksson U. G., Brasch R. C., Tozer A. T., Drug Metab. Dispos., 15, 155-160 (1987).

9) Iannone A., Bini A., Swartz H. M., Tomasi A., Vannini V., Biochem. Pharmacol., 38, 2581-2586 (1989).

10) Chen K., Morse P. D. 2nd, Swartz H. M., Biochim. Biophys. Acta, 943, $477-484$ (1988).

11) Utsumi H., Muto E., Masuda S., Hamada A., Biochem. Biophys. Res. Commun., 172, 1342-1348 (1990).

12) Rizzi C., Samouilov A., Kutala V. K., Parinandi N. L., Zweier J. L., Kuppusamy P., Free Radic. Biol. Med., 35, 1608-1618 (2003).

13) Golman K., Petersson J. S., Ardenkjaer-Larsen J.-H., Leunbach I., Wistrand L.-G., Ehnholm G., Liu K., J. Magn. Reson. Imaging, 12, 929-938 (2000)

14) Krishna M. C., English S., Yamada K., Yoo J., Murugesan R., Devasahayam N., Cook J. A., Golman K., Ardenkjaer-Larsen J. H., Subramanian S., Mitchell J. B., Proc. Natl. Acad. Sci. U.S.A., 99, 22162221 (2006).

15) Elas M., Ahn K. H., Parasca A., Barth E. D., Lee D., Haney C., Halpern H., J. Clin. Cancer Res., 12, 4209-4217 (2006).

16) Bobko A. A., Dhimitruka I. D., Zweier J. L., Khramtsov V. V., J. Am. Chem. Soc., 129, 7240-7241 (2007).

17) Dang V., Wang J., Feng S., Buron C., Villamena F. A., Wang P. G., Kuppusamy P., Bioorg. Med. Chem. Lett., 17, $4062-4065$ (2007).

18) Meenakshisundaram G., Eteshola E., Blank A., Lee S. C., Kuppusamy P., Biosens. Bioelectron., 25, 2283-2289 (2010).

19) Sostaric J. Z., Pandian R. P., Bratasz A., Kuppusamy P., J. Phys. Chem. $B, 111,3298-3303$ (2007).

20) Bangham A. D., Standish M. M., Watkins J. C., J. Mol. Biol., 13, $238-252(1965)$

21) Takeshita K., Utsumi H., Hamada A., Biophys. J., 52, 187-197 (1987).

22) Untrach S. H., Shipley G. G., J. Biol. Chem., 252, 4449—4457 (1977).

23) Jost P., Libertini L. J., Hebert V. C., Griffith O. H., J. Mol. Biol., 59, $77-98$ (1971).

24) Cogan U., Shinitzky M., Weber G., Nishida T., Biochemistry, 12, $521-528$ (1973).

25) Shinitzky M., Barenholz Y., J. Biol. Chem., 249, 2652-2657 (1974).

26) Marsh D., Watts A., "Liposomes: From physical Structure to Therapeutic Applications," Chap. 6, ed. by Knight C. G., Elsevier, Amsterdam, 1981, pp. 139-188.

27) Maspoch D., Catala L., Gerbier P., Ruiz-Molina D., Vidal-Gancedo J., Wurst K., Rovira C., Veciana J., Chem. Eur. J., 8, 3635-3645 (2002).

28) Armet O., Veciana J., Rovira C., Riera J., Castafier J., Molins E., Rius J., Miravitlles C., Olivella S., Brichfeus J., J. Phys. Chem., 91, 56085616 (1987).

29) Bowman M. K., Mailer C., Halpern H. J., J. Magn. Reson., 172, 254 $267(2005)$

30) Kutala V. K., Villamena F. A., Ilangovan G., Maspoch D., Roques N., Veciana J., Rovira C., Kuppusamy P., J. Phys. Chem. B, 112, 158-167 (2008).

31) Oldfield E., Chapman D., Biochem. Biophys. Commun., 43, 610-616 (1971).

32) Shah D. O., Schulman J. H., J. Lipid Res., 8, 215-226 (1967).

33) Inoue K., Biochim. Biophys. Acta, 339, 390- 402 (1974). 